THERMAL IONIZATION ENERGY OF

LITHIUM AND LITHIUM-OXYGEN COMPLEXXES

IN SINGLE-CRYSTAL SILICON

By

Roger Keith Crouch

Thesis submitted to the Graduate Faculty of the

Virginia Polytechnic Institute

in candidacy for the degree of

MASTER OF SCIENCE

in

PHYSICS

August 1967 
THERMAL IONIZATION ENERGY OF

LITHIUM AND IITHIUM-OXYGEN COMPLEXES

IN SINGLE-CRYSTAL SILICON

by

Roger Keith Crouch

Thesis submitted to the Graduate Faculty of the

Virginia Polytechnic Institute

in candidacy for the degree of

MASTEER OF SCIENCE

in

PHYSICS

Approved:

T. E. Gilmer, Jr., Chairman

A. K. Furr

C. D. Williams

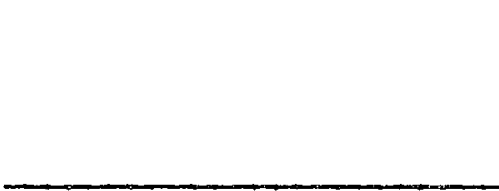

$\sqrt{\mathrm{J} \cdot \mathrm{A} \cdot \mathrm{Jacob} \mathrm{s}}$

August 1967

Blacksburg, Virginia 
TABLE OF CONTENTS

CHAPTER

PAGE

TITLE • • . . . . . . . . . . . . . . . . 1

TABLE OF CONTENTS • • • • • • • • • • • • • • . 2

LIST OF FIGURES . • . . . . . . . . . . . . 3

LIST OF SYMBOLS . . . . . . . . . . . . . . 4

I. INTRODUCTION . . . . . . . . . . . . . . 6

II. DESCRIPTION OF THE EXPERIMFNT . • . • • . • . • . 7

Silicon Crystals . . . . . . . . . . . . 7

Hall Measurements . . . . . . . . . 8

Calculations .................. 10

III. RESULTS AND DISCUSSION . . • . . . . . . . . . 17

Pulled-Crucible Sample . . . . . . . . . . 17

Float-Zone Sample . . . . . . . . . . . . 19

IV. CONCLUDING REMARKS . . . . . . . . . . . . . . 21

V. ACKNOWLEDGMENTS . . . . . . . . . . . . . 22

REFERENCES •. • . . . . . . . . . . . 23

VITA ..................... 25 
IIST OF FIGURES

FIGURE

PAGE

1. Carrier concentration as function of temperature for LiO impurity . . . . . . . . . . . . . 26

2. Effect of consideration of upper level ground state for LiO data................ . . . 27

3. Carrier concentration as a function of temperature for Li impurity . . . . . . . . . . . . . . 28

4. Carrier concentration as a function of temperature for Ii impurity neglecting ground state degeneracy . . . 
LIST OF SYMBOLS

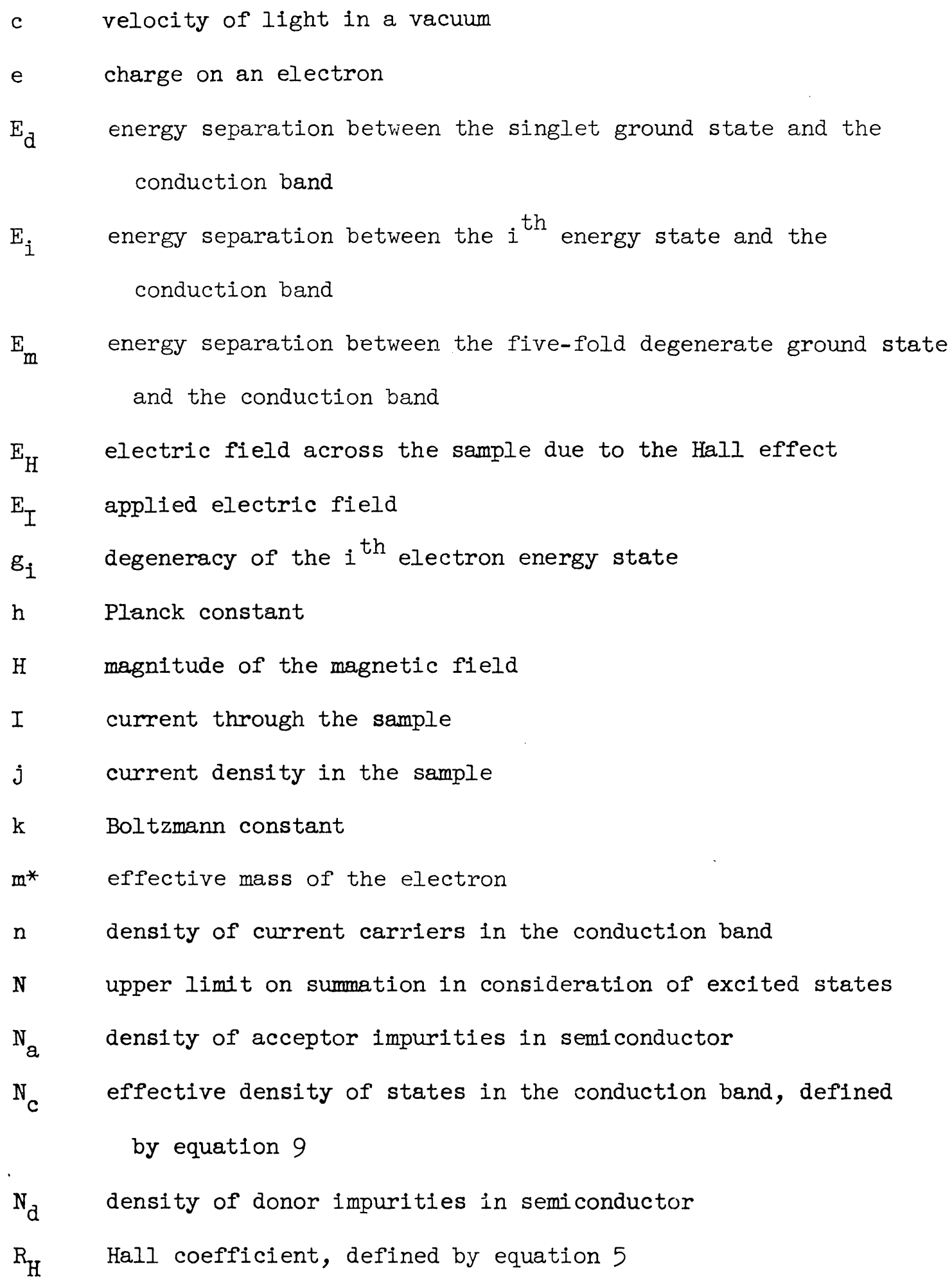


$t$ thickness of rectangular Hall sample

T temperature

$v \quad$ velocity of electrons in direction of current flow

$\mathrm{V}_{\mathrm{H}} \quad$ voltage across sample due to Hall effect

w width of rectangular Hall sample

$\Delta \quad$ energy splitting of ground state of impurity, $E_{m}-E_{d}$

$\Delta_{i} \quad$ energy difference in singlet ground state and $i^{\text {th }}$ energy level,

$$
E_{1}-E_{d}
$$

$\theta \quad$ Hall angle, defined by equation 1

$\mu \quad$ mobility of electron

$\sigma \quad$ conductivity of the material

Is, $2 \mathrm{~s}, 2 \mathrm{p}_{ \pm}$, and so forth, correspond to the optical designations of the energy states of an electron. 
I. INTRODUCTION

The properties of lithium as an impurity in the elemental semiconductor silicon are of great interest due to an increasing number of electronic devices which use lithium doped silicon as a basic material. Optical studies ${ }^{1,2}$ have recently shown that the interstitial lithium impurity exhibits an excitation spectrum which is similar to that exhibited by a Group V impurity; however, the energy levels of this spectrum are dependent upon the amount of oxygen present in the crystal, with which the lithium is known to interact ${ }^{3}$. Measurements of the thermal ionization energy of the lithium impurity is silicon have been made by a group from Bell Laboratories ${ }^{4}$ on a sample which almost certainly contained a large amount of oxygen, yet, the values they obtained agree closely with values determined optically for material which had a low oxygen content. This study deals with a determination of the thermal ionization energy of the lithium impurity in a float-zone crystal (low oxygen content) and a pulled-crucible crystal (high oxygen content), and a comparison of these values with those determined by the optical studies. The thermal data is analyzed taking the splitting of the degenerate ground state into account. It is found that failure to consider this splitting can lead to significant errors in values for ionization energy as well as donor and acceptor concentrations. 
II. DESCRIPTION OF THE EXPERTMENT

\section{Silicon Crystals}

Pulled-crucible crystal.- Silicon crystals grown by the Czochralski or pulled-crucible method typically have on the order of $10^{18}$ atoms $/ \mathrm{cm}^{3}$ of oxygen as an impurity. Kaiser and Keck ${ }^{5}$ have given values of the absorption coefficient at the nine micron band as a function of oxygen concentration. Using their values, the oxygen content of the pulled-crucible sample used in these tests was $5 \times 10^{17}$ atoms $/ \mathrm{cm}^{3}$. The major active impurity prior to doping was boron. The sample resistivity measured on a four-point probe was approximately $5000 \mathrm{ohm}-\mathrm{cm}$, indicating an acceptor concentration of approximately $10^{12} \mathrm{~cm}^{-3}$. The major inert impurity is believed to be carbon having a concentration of a few parts per million.

Float-zone crystal.- Float-zone refined silicon crystals have much lower oxygen concentration than the pulled-crucible crystal. The oxygen content of the float-zone sample used in these tests was not detectable by infrared techniques but is estimated by the manufacturer to be on the order of $10^{15} / \mathrm{cm}^{3}$. The major active impurity was again boron; and the resistivity was $10 \mathrm{ohm}-\mathrm{cm}$ giving an acceptor concentration of $10^{15}$ per $\mathrm{cm}^{-3}$. Also, there were probably a few parts per million of carbon present.

Doping procedure.- The samples were doped using essentially the same technique used by Aggarwal et ${ }^{2}{ }^{2}$. Silicon wafers were coated with a commercially avallable suspension of lithium in mineral oil. The wafers were then heated for 2 hours in a helium environment at 
$200^{\circ} \mathrm{C}$ to drive off the oil. The temperature was raised to $400^{\circ} \mathrm{C}$ and held for 30 minutes to drive the lithium into the silicon crystal. The wafers were then removed from the furnace and the excess lithium cleaned off before being heated in a helium environment at $600^{\circ} \mathrm{C}$ for 2 hours to assure a homogeneous distribution of the lithium and anneal any "thermal conversion" of the type described by Fuller and Logan. 6

\section{Hall Measurements}

Sample preparation ${ }^{7}$ - Standard "bridge-shaped" samples were cut from the doped silicon wafers with an ultrasonic impact grinder. Surface contaminants were removed by etching the samples in CP-4 (a mixture of hydrofluoric, nitric, and acetic acid). The tabs were then sandblasted and plated with nickel by an electrodeless process. A tesla coil was used on each tab to aid in removing any rectifying contacts which may have been present. Indium solder was used to connect the sample to the measuring circuitry of the cryostat.

Measuring system.- The cryostat was similar in design to that described in reference 8 , using liquid helium as a coolant and gaseous helium in the sample chamber as a transfer gas to insure uniform temperatures. Temperatures below $100^{\circ} \mathrm{K}$ were measured by a germanium resistance thermometer, while temperatures greater than $100^{\circ} \mathrm{K}$ were measured by a copper-constantan thermocouple. All voltages were measured on a precision digital voltmeter except those instances where the circuit resistance became comparable to the resistance of the 
voltmeter. In these cases, a potentiometer or an electrometer was used. The magnet provided a uniform field of 4000 gauss over the entire sample for all $\mathrm{Hall}$ measurements.

In measurements of the Hall voltage, certain associated effects give rise to potentials which must be corrected in order to avoid error in the measured value. The largest of these effects is the potential which appears because of the difficulty in alining the measuring probes or tabs directly across the sample. This misalinement results in an "IR drop" or voltage because the two probes are not on the same equipotential, giving a constant error to the Hall voltage. In addition to the IR drop, there are three thermomagnetic or galvano-magnetic effects: the Ettingshausen effect, the Nernst effect, and the Righi-Leduc effect. These effects will give rise to a temperature gradient or a potential gradient when either an electric current or a thermal current is subjected to a magnetic field perpendicular to the direction of current flow. A temperature gradient as well as potential gradient will cause an error in Hall effect measurements. If a series of four measurements is taken reversing the magnetic field and the sample current in all possible combinations, all of the undesired associated effects except the Ettinghausen effect can be eliminated or minimized by taking an average (using the appropriate signs) of the four readings. 9 This procedure was used in these tests. (The Ettinghausen effect was negligible for the samples used in this paper.) 
Calculations

Hall coefficient.- When a magnetic field is applied to a current carrying substance in a direction perpendicular to the direction of the current, a potential gradient will appear across the sample in a direction perpendicular to the plane of the current and the magnetic field. This is the Hall effect and the voltage is the Hall voltage. If the particle nature of conduction is considered, the Hall effect can be explained in the following way: When not under the influence of the magnetic field, the current consists of streams of charged particles drifting longitudinally under the influence of the electric field in the sample. As the magnetic field is applied, the Lorentz force, $\frac{e}{c}(\vec{v} \times \vec{H})$, acts on the electrons; and they are swept to the edge of the sample, building up a field such that at steady-state conditions, the force which this field exerts on the current carrier is equal in magnitude but opposite in sign to the Lorentz force. The presence of this field causes the direction of the electric field vector to differ from that of the current vector by an angle $\theta$, defined by the equation:

$$
\tan \theta=\frac{E_{H}}{E_{I}}
$$

where $E_{H}$ is the field due to Hall effect and $E_{I}$ is the applied field. From the balance of forces at steady-state we may write:

$$
\mathrm{E}_{\mathrm{H}}=\frac{1}{\mathrm{c}} \mathrm{vH} \text {, }
$$

assuming that the current and magnetic field are perpendicular to each other. 
In most cases the field due to Hall effect is quite small compared to the applied field and the tangent of $\theta$ can be approximated by $\theta$. Thus, combining (I) and (2) we have:

$$
\theta=\frac{E_{H}}{E_{I}}=\frac{v H}{c E_{I}}=\frac{\mu H}{c}
$$

where $\mu$ is the mobility of the current carrier. Since $E_{H}$ is small, we may write $j=\sigma E_{I}$, where $j$ is the current density and $\sigma$ is the conductivity of the material. Thus, we obtain the relationship

$$
E_{H}=\theta E_{I}=\left(\frac{\mu}{\sigma c}\right) H j
$$

The Hall coefficient is defined as the quantity in parentheses on the right side of equation (4), that is,

$$
\mathrm{R}_{\mathrm{H}}=\frac{\mu}{\sigma \mathrm{c}}=\frac{1}{\mathrm{nec}}
$$

where $\sigma$ is given by $\sigma=$ ne $\mu$ and $n$ is the density of current carriers. Therefore, we see by this simplified analysis that the Hall coefficient is inversely proportional to the number density of electrons in the conduction band for an n-type semiconductor. As stated, this is an oversimplified expression for the true relationship between the Hall coefficient and the electron concentration due to the differences In the mobility used in equation (3) and the mobility required in the expression for the conductivity. Consideration of a Boltzmann distribution for the velocities of the electrons caused by scattering due to thermal vibrations of the crystal lattice leads to a factor of $\frac{3 \pi}{8}$ in equation (5)..$^{10}$ However, for temperatures less than about 
$100^{\circ} \mathrm{K}$, Long and Myers have shown that ionized impurity scattering is important and that the proportionality term between $R_{H}$ and $n$ is a complex function of temperature and concentration. ${ }^{11}$ Since the proportionality term is never too different from unity, it is assumed to be 1.0 for all calculations in this paper.

In order to calculate the Hall coefficient from the measured quantities: $\mathrm{V}_{\mathrm{H}}$, the Hall voltage; I, the current through the sample; $w$, the width of the sample; and $t$, the thickness of the sample, we write equation (4) in the form

$$
\frac{\mathrm{V}_{\mathrm{H}}}{\mathrm{W}}=\mathrm{R}_{\mathrm{H}} \times \mathrm{H} \frac{\mathrm{I}}{\mathrm{wt}}
$$

or

$$
\mathrm{R}_{\mathrm{H}}=\frac{\mathrm{v}_{\mathrm{H}} \mathrm{t}}{\mathrm{HI}} \times 10^{8}
$$

where the factor of $10^{8}$ comes from a conversion to practical units from c.g.s units and absolute e.s.u. and e.m.u. ${ }^{12}$ Values for $n$ were determined from equation (5) modified to the appropriate units.

Ionization energy.- The equation most widely used in the analysis of Hall effect data ${ }^{13}$ can be written as:

$$
\frac{n\left(n+N_{a}\right)}{N_{d}-N_{a}-n}=N_{c} \exp \left(\frac{-E_{d}}{k T}\right)
$$

where $\mathrm{N}_{\mathrm{d}}$ is the concentration of donor impurities per $\mathrm{cm}^{3}, \mathrm{~N}_{\mathrm{a}}$ is the concentration of acceptor impurities per $\mathrm{cm}^{3}, \mathrm{~N}_{c}$ is the effective density of states in the conduction band defined by 


$$
\mathbf{N}_{c}=2\left(\frac{2 \pi \mathrm{m}^{*} \mathrm{kT}}{\mathrm{h}^{2}}\right)^{3 / 2}
$$

$\mathrm{m}^{*}$ is the effective mass of the electron, $k$ is Boltzmann's constant, $h$ is Planck's constant and $E_{d}$ is the ionization energy (positive) of the donor electron.

However, this equation fails to consider the presence of any excited impurity states. Kohn and Luttinger have calculated the type of energy level scheme which a substitutional donor impurity would be expected to have, taking into account the known structure of the bottom edge of the conduction band.14,15 By solving the effective-mass equation, energy levels of the ground and excited states of the impurity were found; and in analogy with the hydrogen atom, these states were designated as the "ls," "2s," "2p," and so forth. Since the wave function for the effective mass "Is" state was constructed from the Bloch functions of the six equivalent minima of the conduction band, it would be six-fold degenerate. Since the theoretical values for the effective mass ground state energy level did not agree with the experimental value for the ionization energy of various donor impurities, but the predicted levels for the various excited states agreed closely with the experimental infrared data, Kohn and Luttinger pointed out that the effective mass theory was not applicable in the region rather close to the donor ion due to the failure of some of the assumptions, mainly, the form of the potential. However, calculations of the so-called valley-orbit splitting ${ }^{15}$ show that the six-fold degenerate ground state is split into three levels 
with degeneracies of 1,2 , and 3 . The singlet state has the lowest energy for a group $\mathrm{V}$ impurity and calculations considering the valleyorbit splitting give ionization energies that agree very well with the experimental values. ${ }^{16}$ The doublet and triplet states are relatively close in energy and can normally be considered to be equivalent to a five-fold degenerate level, close to the predicted value for the effective mass ground state energy.

Using an energy level structure similar to that predicted by Kohn and Luttinger, the distribution of electrons among all the states of the conduction band, and any compensating impurities is given by the statistical equation:

$$
\frac{n\left(n+N_{a}\right)}{N_{d}-N_{a}-n}=\frac{N_{c}}{\sum_{i=1}^{N} g_{i} \exp \left(E_{i} / k T\right)}
$$

where $g_{i}$ is the degeneracy of the $i^{\text {th }}$ level and $E_{i}$ is the energy separation between the $i^{\text {th }}$ level and the conduction band. ${ }^{17}$ The upper limit of the sumation is limited to a finite value since after the first few excited states, the rest would be indistinguishable from the conduction band. For silicon, equation (10) may be written as:

$$
\frac{n\left(n+N_{a}\right)}{N_{d}-N_{a}-n}=\frac{2.75 \times 10^{15} \exp \left(\frac{-E_{d}}{k T}\right)}{1+5 \exp \left(\frac{\Delta}{k T}\right)+\sum_{i=2}^{N} g_{i} \exp \left(\Delta_{i} / k T\right)}
$$

where the appropriate value for the effective density of states has been used in the form $2.75 \times 10^{15} \mathrm{~T}^{3 / 2}, \mathrm{E}_{\mathrm{d}}$ is the energy of the 
singlet ground state, $\Delta=E_{m}-E_{d}$ where $E_{m}$ is the energy of the five-fold degenerate ground state, and $\Delta_{i}=E_{1}-E_{d}$. It is seen that at high enough temperatures such that essentially all uncompensated donors are ionized, $\mathrm{n}$ is given by $\mathrm{N}_{\mathrm{d}}-\mathrm{N}_{\mathrm{a}}$. At very low temperatures, $\mathrm{n}<<\mathrm{N}_{\mathrm{a}}$ so that equation (II) may be written as:

$$
\frac{n}{T^{3 / 2}}=\frac{N_{d}-N_{a}}{N_{a}} \frac{2.75 \times 10^{15} \exp \left(\frac{-E_{d}}{k T}\right)}{1+5 \exp \left(\frac{\Delta}{k T}\right)+\sum_{i=2}^{N} g_{i} \exp \left(\triangle_{i} / k T\right)}
$$

Invariably, $\Delta_{i}<0$ such that at very low temperatures, the summation may be ignored. Similarly, if $\Delta<0$, as it normally is, equation (12) for low enough temperatures can be written in the form:

$$
\ln \left(\mathrm{n} / \mathrm{T}^{3 / 2}\right)=\ln \left[\frac{2.75 \times 10^{15}\left(\mathrm{~N}_{\mathrm{d}}-\mathrm{N}_{\mathrm{a}}\right)}{\mathrm{N}_{\mathrm{a}}}\right]-\mathrm{E}_{\mathrm{d}} / \mathrm{kT}
$$

therefore, the slope of a plot of $2 \mathrm{n} \mathrm{n} / \mathrm{T}^{3 / 2}$ as a function of $I / T$ will yield a value for the ionization energy of the donor impurity. This is identical to the result obtainable from equation (8). However, if the temperature is not low enough to ignore the term involving $\Delta$, the ionization energy of donor impurity can be found from an equation of the form: ${ }^{11}$

$$
\mathrm{E}_{\mathrm{d}}=\frac{\mathrm{k}}{\left(1 / \mathrm{T}_{2}-1 / \mathrm{T}_{1}\right)}\left\{\ln \left(\frac{\mathrm{n}_{1} / \mathrm{T}_{1}^{3 / 2}}{\mathrm{n}_{2} / \mathrm{T}_{2}^{3 / 2}}\right)+\ln \left[\frac{1+5 \exp \left(\Delta / \mathrm{kT}_{1}\right)}{1+5 \exp \left(\Delta / \mathrm{kT}_{2}\right)}\right]\right\}
$$

where the subscripts 1 and 2 correspond to experimental values at temperatures where $\mathrm{n}<<\mathrm{N}_{\mathrm{a}}$. Using values of $\mathrm{N}_{a}$ determined from the low temperature equation 


$$
N_{a}=\frac{N_{d}-N_{a}}{n} \frac{2.75 \times 10^{15} \mathrm{~T}^{3 / 2} \exp \left(\frac{-E_{d}}{k T}\right)}{1+5 \exp (\Delta / \mathrm{kT})}
$$

Long and Myers ${ }^{18}$ determined the optimum value of $\Delta$ to give a best fit of equation (1I) to experimental Hall data which they had obtained. They found $\Delta$ to be between 9-12 meV for phosphorous. Aggarwal ${ }^{19}$ later made optical measurements of the splitting which agree very well with the thermal data, being $11.85 \mathrm{meV}$. (Aggarwal found that the doublet and triplet state in phosphorous were split by $1.35 \mathrm{meV}$, the triplet state having the lowest energy.)

The data presented in this paper were analyzed by means of a computer program which fitted equation (II) to the experimental data by the method of least squares with respect to the three parameters $N_{d}, N_{a}$, and $E_{d}$. Comparison of values calculated using equations (14) and (1.5) and values calculated by the computer program for phosphorous data showed very good agreement (differences around 0.5 percent for $E_{d}$ and 5 percent for $N_{a}$ ). These calculations also showed that consideration of the excited states other than the upper ground states made essentially no difference in the results. Appropriate values of $\Delta$ are a required input of the program and were obtained from the literature. 
III. RESULTS AND DISCUSSION

\section{Pulled-Crucible Sample}

Figure 1 presents a plot of the carrier concentration as a function of temperature for the pulled-crucible sample. (This impurity has been previously identified as LiO. ${ }^{1,2}$ ) The curve in the figure corresponds to the values predicted by the computer program using $\Delta=-7.7 \mathrm{meV} .^{2}$ The values for $E_{d}, N_{a}$, and $N_{d}$ from the computer program were $37.3 \mathrm{meV}, 2.17 \times 10^{12} \mathrm{~cm}^{-3}$ and $3.74 \times 10^{15} \mathrm{~cm}^{-3}$, respectively. The values for $\mathrm{N}_{\mathrm{d}}$ agree with values of $\mathrm{n}$ in the region where nearly all uncompensated donors are ionized, viz. $3.67 \times 10^{15} \mathrm{~cm}^{-3}$, and $N_{a}$ is in agreement with the acceptor concentration prior to doping $\left(2.6 \times 10^{12} \mathrm{~cm}^{-3}\right)$. The value for the ionization energy is slightly lower than the optical value of $39 \mathrm{mev},{ }^{1}$ but seems to agree with the optical value much better than the thermal value of $33 \mathrm{meV}$ reported by Morin et al. ${ }^{4}$ Using the values obtained from the computer program for $\mathrm{N}_{d}, \mathrm{~N}_{a}$, and $\mathrm{E}_{d}$, figure 2 shows the effect which consideration of the upper ground state has on the fit of the curve to the experimental data. The curve labeled $\Delta=-\infty$ is simply equation (8), whereas the curve labeled $\Delta=-7.7 \mathrm{meV}$ is equation (14), neglecting the sumation terms. It is apparent that the upper level of the ground state affects the shape of the curve over an intermediate temperature range.

The difference between the present thermal value for the 1ontzation energy and the optical value, as well as the difference in the present and previous thermal value can, at least partially, be 
explained by a consideration of the excited impurity states. Kohn commented about the difference in thermal and optical values for ionization energy but offered no explanation. ${ }^{15}$ Various papers have noted a difference in thermal ionization energy as a function of concentration. ${ }^{11,21}$ This dependency of $E_{d}$ upon concentration appears to be fairly well explained by the model proposed by Erginsoy ${ }^{21}$ and elaborated somewhat by Baltensperger 22 in which the excited impurity states form bands which overlap with the conduction band, effectively lowering the conduction band edge. An increase in concentration would result in more overlap for a lower excited state; thus, the conduction band would be lowered even farther. It is believed that this model could also aid in explaining the differences between optical and thermal values. The optical value for $E_{d}$ is determined by adding the value calculated by effective mass theory for the depth of an excited state to the optically determined value for a transition from the ground state to the excited state. ${ }^{15}$ The excited state used is generally the $3 p_{ \pm} \cdot 1,2$ The effect of the overlap of the higher order states would not be seen except in the disappearance of such states as the $6 p_{ \pm}, 5 p_{ \pm}, 4 p_{ \pm}$, and so forth, from the optical spectra as the concentration is increased. Eventually, the $3 p_{ \pm}$peak would itself disappear; however, before this point is reached, the number of free carriers still in the conduction band even at these lower temperatures 1s usually such that for a reasonably thick sample, most of the infrared radiation is absorbed by the sample, and resolution of the absorption peaks becomes very difficult. Therefore, other than for 
some coricentration broadening of the peaks, the optical ionization energy would generally be unchanged for different impurity concentrations. Thus, it seems that this model would partly explain the discrepancy between the thermal value obtained here and optical values. Another possibility for the difference in thermal and optical values of Iithium in silicon could be occupation by electrons of energy levels close to that identified as a LiO state but having a slightly higher energy. Gilmer, Franks, and Bell identified six donor-like series of spectra in a sample similar to the one in this paper. ${ }^{I}$ If these were possible electron states, their energies could not be separated by Hall measurements, and the ionization energy deduced from the Hall data would be a weighted average of the energy of each of these states. Since they would have a lower ionization energy than the primary state at $39 \mathrm{meV}$, the resultant thermal ionization energy would be smaller.

\section{Float-Zone Sample}

The carrier concentration as a function of temperature for the float-zone sample is presented in figure 3. (This impurity has been identified as an isolated lithium atom. $)^{1,2}$ The solid line corresponds to the fitted curve from the computer program using $\Delta=1.6 \mathrm{mev} .^{2}$ Calculated values of $\mathrm{N}_{\mathrm{d}}$ and $\mathrm{N}_{\mathrm{a}}$ were $1.08 \times 10^{17} \mathrm{~cm}^{-3}$ and $7.4 \times 10^{14} \mathrm{~cm}^{-3}$, respectively, which compare quite well with the experimentally determined values of $9.9 \times 10^{16} \mathrm{~cm}^{-3}$ and $10^{15} \mathrm{~cm}^{-3}$ for $N_{d}$ and $N_{a}$. The value for $E_{d}$, the energy separation between the singlet ground state and the conduction band, was $27.9 \mathrm{meV}$. The curve in figure 3 was calculated using the structure of the ground 
state which Aggarwal et al. used to explain their optical data. In this model the energy separation between the five-fold degenerate ground state and the conduction band is greater than the energy separation between the singlet ground state and the conduction band; therefore, the ionization energy in this case would be $E_{m}$ or $29.5 \mathrm{meV}$, from the relationship $\mathrm{E}_{\mathrm{m}}=\mathrm{E}_{\mathrm{d}}+\Delta$. (This "anomalous" ground state structure could be due to the interstitial location of lithium.) This value of $29.5 \mathrm{MeV}$ seems to be comparable to the optical value of $32 \mathrm{meV},{ }^{1,2}$ particularly in view of the relatively high impurity concentration of this sample. The agreement between the calculated values and the experimental data using the model proposed by Aggarwal et al. seems to imply that their description of the ground state stmucture is adequate for this type of study.

Consideration of the splitting of the degenerate ground state is essential in the analysis of the lithium data. Fallure to consider this degeneracy results in errors in the values obtained by the computer program for $E_{d}, N_{d}$, and $N_{a}$, and a resultant poor fit of predicted to measured values of $n$. Figure 4 presents a plot of the carrier concentration as a function of temperature where the solid line represents the least square fit neglecting to consider the splitting of the degenerate ground state. Values for $E_{d}, N_{d}$, and $N_{a}$ were $25.9 \mathrm{meV}$, $5.7 \times 10^{16} \mathrm{~cm}^{-3}$, and $1.7 \times 10^{16} \mathrm{~cm}^{-3}$, respectively. Thus neglecting the splitting gives a value of $N_{d}$ that is in error by about a factor of 2 , an $\mathrm{N}_{a}$ that is an order of magnitude higher than it should be compared to predope resistivity measurements, and an ionization energy that is too small by about 12 percent. 
IV. CONCI,UDING REMARKS

Measurements of the thermal ionization energy of lithium impurities in single-crystal silicon by means of the Hall effect have shown that the ionization energy is a function of the oxygen concentration in the silicon. Values determined were $29.5 \mathrm{meV}$ for a floatzone crystal (believed to have about $10^{15}$ oxygen atoms per $\mathrm{cm}^{3}$ ) and $37.3 \mathrm{meV}$ for a pulled-crucible crystal (having about $5 \times 10^{17}$ oxygen atoms per $\mathrm{cm}^{3}$ by infrared measurements) which tend to agree with values previously determined optically. These measurements also showed the importance of considering the degeneracy of the ground state in calculating the ionization energy, particularly in the float-zone crystals where the splitting of the ground state is relatively small. The ground state structure proposed by Aggarwal et al., wherein the five-fold degenerate state has a lower energy than the singlet state, seems to adequately describe the data. 


\section{ACKNOWLEDGMENTS}

The author wishes to express his appreciation to Dr. T. E. Gilmer, Jr. for his assistance and guidance. The suggestion of this topic as well as assistance in the development of the computer by Dr. R. K. Franks is also greatly appreciated. The help of in sample preparation and equipment construction was very beneficial and the author wishes to thank him. Special recognition and thanks are due the author's wife, , for her encouragement and patience. 
REFERENCES

l. Gi.lmer, T. E., Jr.; Franks, R. K.; Bell, R. J.: J. Phys. Chem. Solids 26, 1195 (1965).

2. Aggarwal, R. I.; Fisher, P.; Mourzine, V.; and Ramdas, A. K.: Phys. Rev. 138, A882 (1965).

3. Pell, E. M.: Solid State Physics in Electronics and

Telecommunications. (Academic Press, New York, 1960), vol. I, p. 261 .

4. Morin, F. J.; Maita, J. P.; Shulman, R. G.; and Hannay, N. B.: Phys. Rev. 96, 833 (1953).

5. Kalser, W.; and Keck, P. H.: J. Appl. Phys. 28, 882 (1957).

6. Fuller, C. S.; and Iogan, R. A.: J. Appl. Phys. 28, 1427 (1957).

7. Breckenridge, R. A.; and Debnam, W. A., Jr.: private communication.

8. Fritzsche, H.: Methods in Experimental Physics, edited by K. Lark-Horovity and Vivian A. Johnson (Academic Press, New York, 1959), vol. 6, part B, p. 157 .

9. Lindberg, Olof: Proc. of I.R.E. 40, 1414 (1952).

10. Shockley, W.: Electrons and Holes in Semiconductors. (D. Van Nostrand Co., Inc., New York, 1950) p. 277.

11. Long, D.; and Myers, J.: Phys. Rev. 115, 1107 (1959).

12. Shockley, op. cit., p. 213.

13. Lee, P. A.: British J. Appl. Phys. 8, 340 (1957).

14. Kohn, W.; and Luttinger, J. M.: Phys. Rev. 98, 915 (1955). 
15. Kohn, W.: Solid State Physics. Edited by F. Seitz, and

D. Turnbull (Academic Press, Inc., New York, 1957), vol. 5, pp. 257-320.

16. Narita, K.; and Shimizer, T.: J. Phys. Soc. Japan 16, 2588 (1961).

17. Blakemore, J. S.: Semiconductor Statistics, (Pergamon Press, New York, 1962), p. 143 .

18. Long, D.; and Myers, J.: Phys. Rev. 115, 1119 (1959).

19. Aggarwal, R. I.: Solid State Comuni cations 2, 163 (1964).

20. Debye, P.; and Conwell, E.: Phys. Rev. 23, 693, (1954).

21. Erginsoy, C.: Phys. Rev. 80, 1104 (1950); Phys. Rev. 88, 893 (1952)

22. Baltensperger, W.: Phil. Mag. (Series 7) 44, 1355 (1953). 


\section{The vita has been removed from the scanned document}




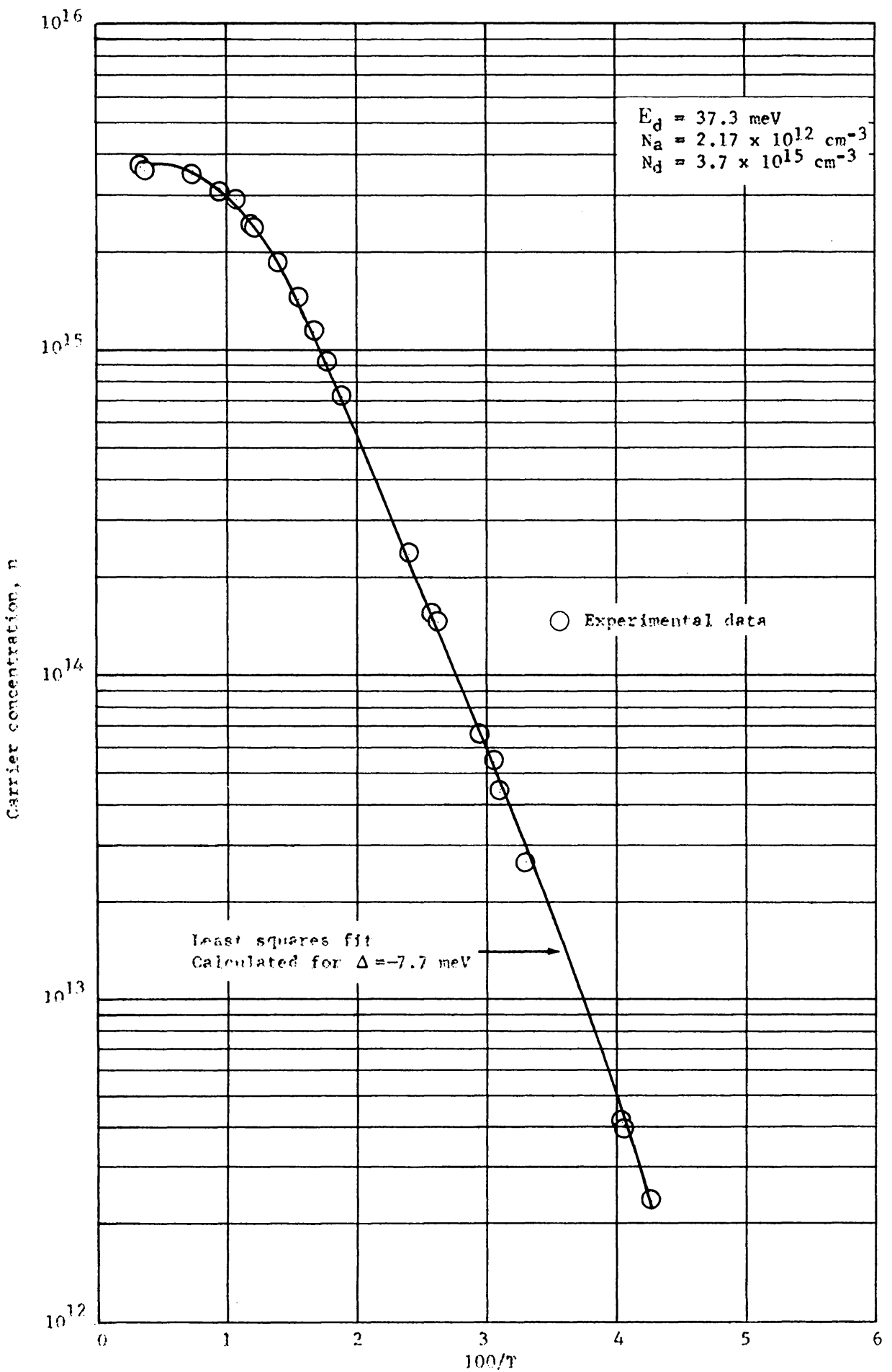

Figure 1.- Carrier concentration as function of temperature for LiO impurity. 


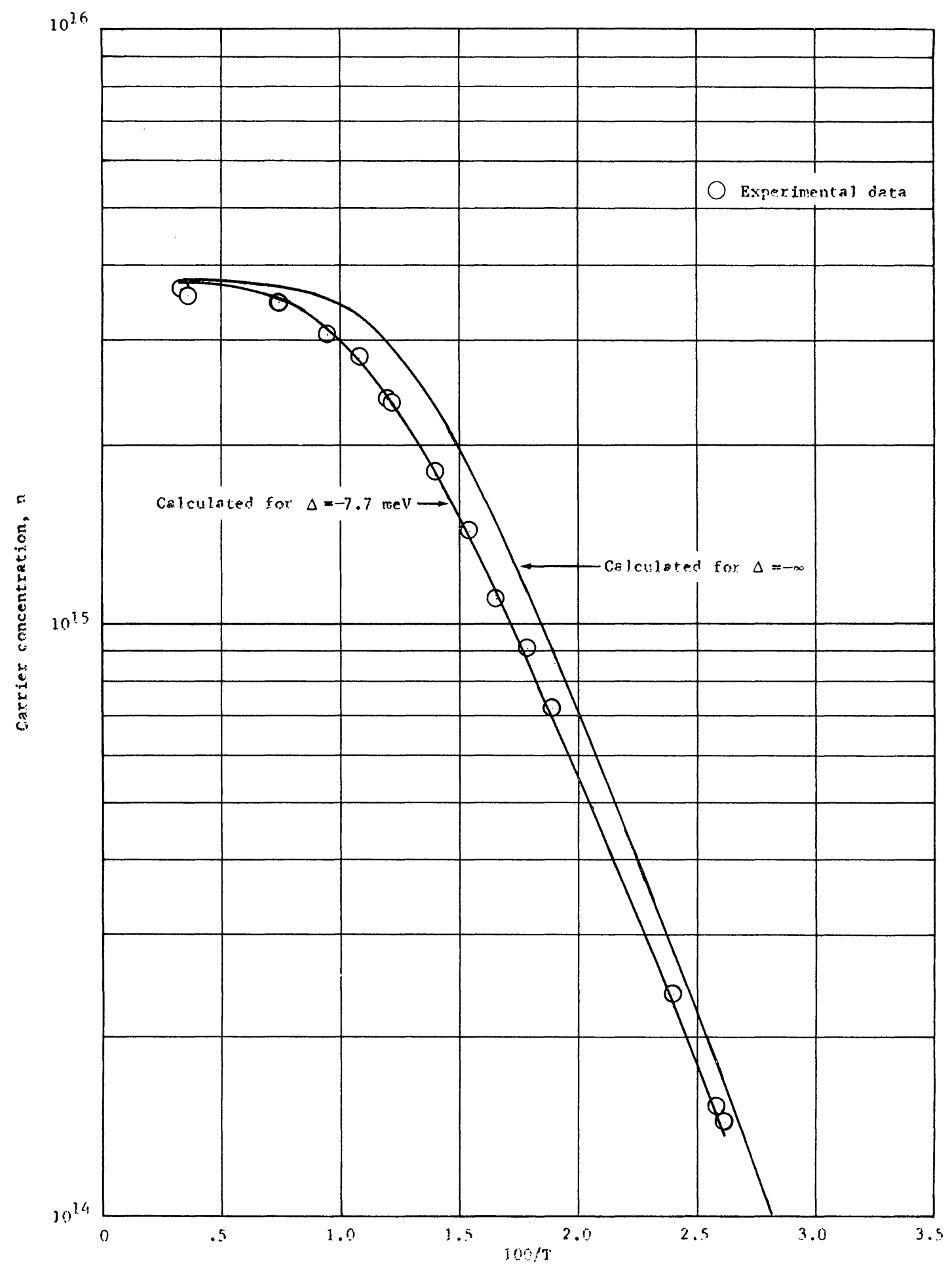

Figure 2.- Effect of consideration of upper level ground state for LiO data. 


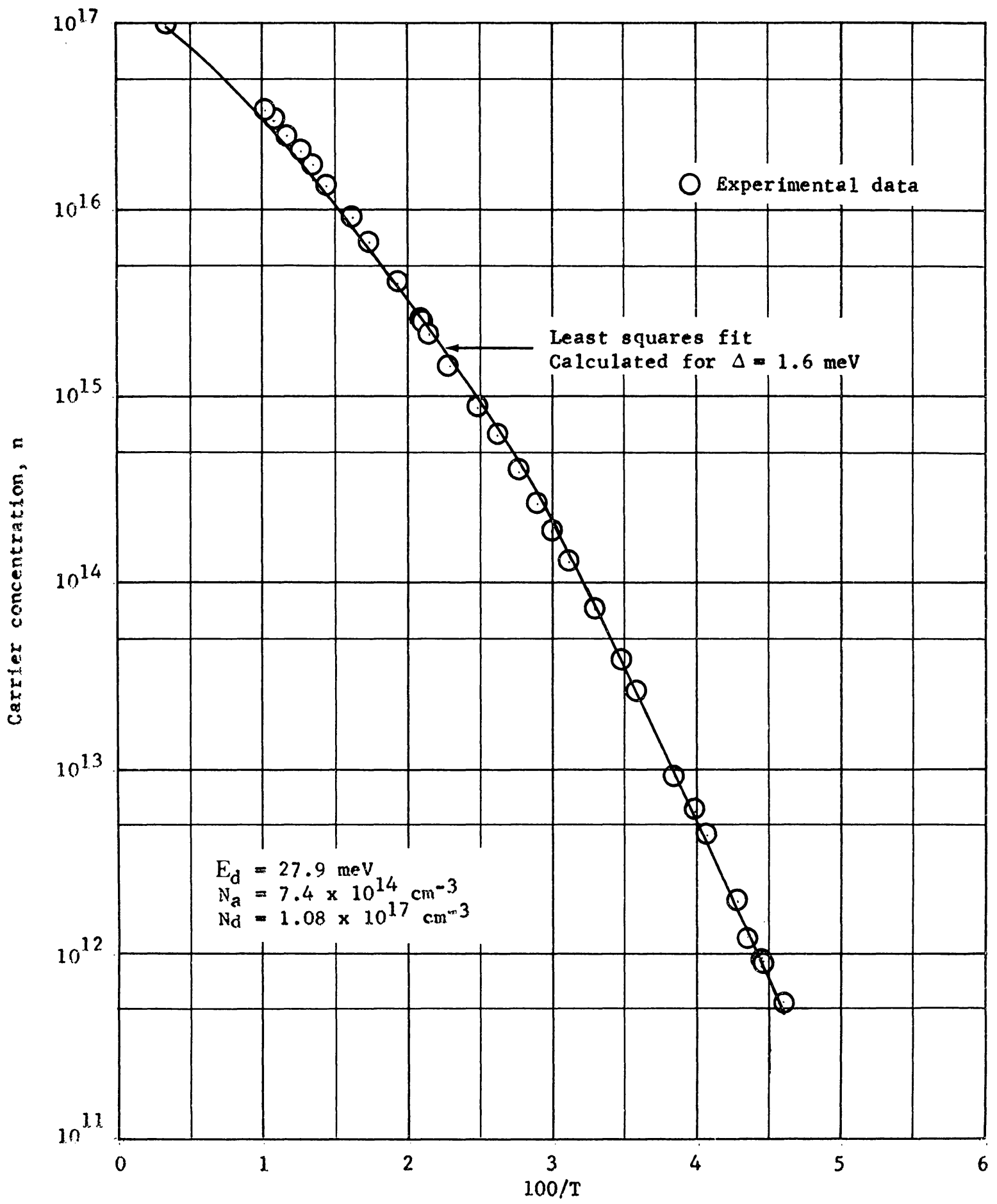

Figure 3.- Carrier concentration as a function of temperature for Li impurity. 


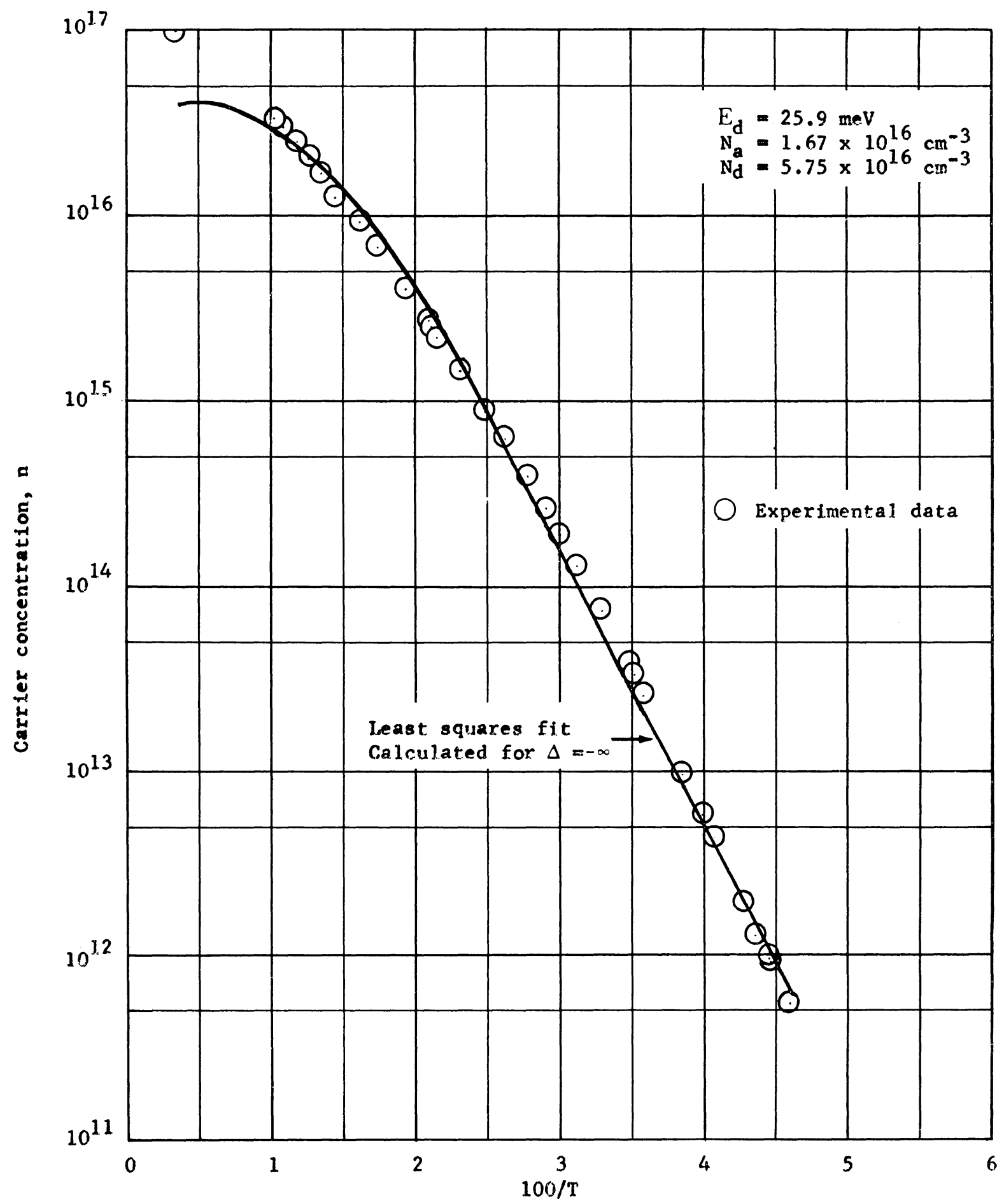

Figure 4.- Carrier concentration as a function of temperature for Li impurity neglecting ground state degeneracy. 


\title{
THERMAL IONIZATION ENERGY OF \\ LITHIUM AND LITHIUM-OXYGEN COMPLEXES \\ IN SINGLE-CRYSTAL SILICON
}

By

Roger Keith Crouch

\begin{abstract}
The ionization energy of lithium as an impurity in single-crystal silicon has recently been determined by optical means to be dependent upon the amount of oxygen in the silicon. It was found that for floatzone crystals, having a relatively low oxygen content (less than $10^{16} \mathrm{~cm}^{-3}$ ), the ionization energy was about $32 \mathrm{meV}$, whereas for a pulled-crucible crystal, having a higher oxygen concentration (approximately $10^{18} \mathrm{~cm}^{-3}$ ), the ionization energy was $39 \mathrm{meV}$. Previous measurements by means of Hall effect studies had indicated that the ionization energy in a pulled crucible sample was about $33 \mathrm{meV}$ which seemed to agree with the optical float-zone value. In an attempt to clarify the situation, Hall effect measurements were made on pulledcrucible and float-zone silicon samples containing lithium as an impurity, and ionization energies were calculated. It was found that this new Hall effect data seemed to show the same dependency on oxygen content as the optical data had shown with the ionization energy, being $37 \mathrm{meV}$ for the pulled crucible sample and $29.5 \mathrm{meV}$ for the float-zone
\end{abstract}


Attention Patron:

2nd page of abstract missing 